\title{
DISEÑO EN LA PORCELANA RUSA DE PRINCIPIOS DEL XX
}

\section{DESIGN IN RUSSIAN PORCELAIN IN THE EARLY 20TH}

\author{
Ekaterina Erokhina \\ Universidad de Málaga \\ ekaterinaerokh@gmail.com
}

Recibido: 02 abril 2020 Aceptado: 20 mayo 2020 Publicado: 01 julio 2020

\begin{abstract}
Resumen: La porcelana soviética vanguardista realizada entre 1918-1923 es una de las más prestigiosas y más caras en el mundo. Es un caso en la Historia del Arte que no tiene precedentes. Esto se debe no sólo a la calidad excepcional de la porcelana, sino también porque los diseños eran más que revolucionarios y glorificaban el país recién nacido. Con este trabajo se pretende hacer un acercamiento a la historia de su producción y relación con el estado y estudio de la porcelana vanguardista soviética.
\end{abstract}

Palabras clave: Porcelana, Soviética, Vanguardia, Suprematismo, Diseño

\begin{abstract}
The avant-garde Soviet porcelain made between 1918-1923 is one of the most prestigious and expensive in the world. It's an unprecedented case in Art History. This is due not only to the exceptional quality of porcelain, but also because of the revolutionary designs that glorified the newborn country. The aim of this work is to approach the history of production, its relationship with the state and study of Soviet avant-garde porcelain.
\end{abstract}

Keywords: Porcelain, Soviet, Avant-Garde, Suprematism, Design.

\section{Introducción}

La porcelana rusa de principios del XX está muy ligada a política y puede considerarse como arte de propaganda. Sin embargo, en este caso la palabra propaganda no es algo peyorativo. Es más, la porcelana artística producida en la fábrica de San Petersburgo debe ser vista como una continuación lógica de toda la vanguardia rusa y no como una consecuencia de la misma Este trabajo pretende un acercamiento a la historia y análisis de algunas obras. Para hacer posible este trabajo, se ha realizado un viaje a la fábrica de 
Porcelana en San Petersburgo, donde también he podido consultar los archivos de la misma.

\section{Historia}

El arte después de la Revolución fue nada más ni nada menos que la transformación de la realidad cotidiana. Nuevas formas debían ser creadas. Una de las primeras cuestiones establecidas después de la Revolución fue la función del arte dentro de la nueva sociedad. Las autoridades soviéticas comprendían muy bien el poder que ejerce el arte sobre la sociedad. Como menciona Lunacharsky (comisario de Narkmporos): "el arte es un medio de educación que tiene un poder colosal. Puede ser que es una de las formas de propaganda más potente que jamás ha existido". (Filatov, 1927, p. 6)

Después de la Revolución de octubre de 1917, la fábrica de porcelana quedó bajo el control del Comisariado del Pueblo de la Educación (Narkompros). Este antiguo equivalente soviético de un ministerio estableció el objetivo de no solo preservar las mejores tradiciones de la porcelana artística rusa, sino también convertir la antigua fábrica de la corte imperial en "un laboratorio de pruebas de cerámica de importancia republicana" (Filatov, 1927, p. 6) para producir porcelana de agitación en el sentido elevado de la palabra: "revolucionaria en contenido, perfecta en forma, impecable en ejecución técnica". Lunacahrsky fijó la tarea de la fábrica en "convertirse en el proveedor de todo el pueblo". (Filatov, 1927, p. 7)

Así mismo, en el 1918 Serguei Chekhonin fue nombrado el director artístico de la planta. Antes de la revolución, era miembro de la asociación El mundo del Arte [MIR ISKUSSTVA] y era conocido como un maestro de la ilustración de libros, un gran conocedor de varios estilos, un conocedor y coleccionista de arte popular. Chekhonin aplicó con éxito su brillante dominio del arte al adorno de la porcelana. Su nombramiento es muy decisivo para historia de la fábrica. Abrió el camino para los experimentos de vanguardia que vamos a ver a continuación.

La porcelana vanguardista es realmente el resultado de más de diez años de crecimiento de la vanguardia rusa. Ni siquiera una consecuencia, sino más bien una 
continuación lógica: los artistas de la fábrica crearon la vanguardia rusa junto con los futuristas, cubistas, constructivistas, suprematistas...

Los primeros años del poder soviético en Rusia estuvieron marcados por una efusión de arte de masas con fines de propaganda. Los productos de la renombrada fábrica estatal de porcelana comenzaron a llevar los mismos eslóganes, citas y aforismos que se podían encontrar en carteles y paneles en las calles y plazas. Todo esto fue parte del plan de la Propaganda Monumental.

Lenin tomó prestada la idea de propagada monumental de la utopía "Ciudad del Sol" de Tomasso Campanella. Se redactó una lista con 69 personajes: revolucionarios, científicos, escritores, músicos. Además, aparece una extensa cita de 28 frases preparada por Narkompros y aceptada por Lenin. Entre otras, encontramos frases de Marx, Cicerón, Ovidio, Tomas Mann, Dostoevsky, Tolstoy y frases sacadas del Nuevo Testamento.

Sin embargo, entre 1917 - 1922 tuvo lugar la Guerra Civil. Pero incluso en los años tan tormentosos, la vida cultural no paraba y se pretendía que algún día esta porcelana flameante fuera a entrar en las casas de todo el pueblo soviético. Entendemos bien que esto nunca se llegó a cumplir por varias razones.

1)La porcelana era muy cara y se producía con tiradas muy pequeñas, no más de 300 ejemplares. Incluso cuando utilizaban imprenta para algunos trabajos, aun así, era muy cara para la mayoría.

2) No solo esto, fue imposible adquirirla realmente porque el estado compraba el 95\% de toda la producción para su venta posterior al extranjero. De esta manera generaban ingresos. Solo existían tres tiendas físicas, una directamente en la fábrica, otra en Moscú y una en Petrogrado (San Petersburgo).

3) La porcelana vanguardista fue el mejor representante de un estado recién nacido: participaba en varias exposiciones internacionales, por ejemplo, en la exposición Universal de París (1925) presentaron 300 trabajos de porcelana, recibieron varios premios y en general gozó de gran éxito en la exposición. Participar en las exposiciones internacionales fue crucial para la U.R.S.S. porque así demostraban su poder artístico a todos aquellos que acusaban la U.R.S.S. en la oposición hacia el 
retroceso en el mundo del arte. Es más, debido al éxito en las exposiciones internacionales, especialmente después de la Exposición en París en 1925 se cimentó la idea de producir, teniendo en cuenta factores primordiales como los intereses de la exportación rentable y la demanda de las empresas interesadas.

Los primeros trabajos de la porcelana vanguardista se producían todavía en los blancos que quedaban en la dispensa de la fábrica. Era porcelana producida durante los reinados de Alejandro II, Alejandro III y Nicolai II. En principio tachaban los sellos de los emperadores, pero al entender el valor histórico que esto representaba en el extranjero, decidieron poner el sello soviético al lado del sello imperial.

Las primeras falsificaciones aparecen ya en el año 1922 cuando la fábrica puso a la venta las piezas en blanco, sin sellos, relativamente baratos. Es esencial saber mirar los sellos para poder asegurar la procedencia de la obra. Si hablamos de la escultura de porcelana hace falta mirar su peso y tamaño. La pieza original pesa más y tiene que ser de mayor tamaño.

\section{Diseño}

Los años 20 son los más prolíficos para historia de la planta. Se produjeron muchos trabajos, la fábrica se extendió, aparecieron nuevos artistas. Uno de los artistas más brillantes de la época fue A.V. Shekatihina-Pototskaya. Shekotihina fue alumna de N. Roerich. Sus obras ignoran todas las leyes de la pintura de porcelana y se caracterizan por la libertad de expresión; son similares a un antiguo lubokı ruso o un icono, al mismo tiempo actualizado y más vivo. Shekotihina-Pototskaya deforma y rompe la perspectiva, construye la composición por sus propias leyes. El dibujo simplificado se combina con detalles delicadamente dibujados en sus obras, con un diseño sofisticado y rico de texturas y fragmentos ornamentales.

A mediados de los años 20, M.V. Lebedeva entró a trabajar en la fábrica. Uno de los temas principales de su obra fue el problema del destino de la persona en las etapas cruciales de la historia; la impresión de los difíciles enfrentamientos de las dos épocas, la

1Un lubok en un tipo de arte popular ruso. Se realizó en la técnica de xilografía, grabado en cobre, litografía 
vieja con la nueva, donde siempre gana la nueva. La mayoría de las historias narradas por Lebedeva son testigos de los acontecimientos que son muy simbólicos por sí mismos. Por ejemplo, "V Aniversario de la Revolución de Octubre" representa la hoguera simbólica, en la que queman el mundo viejo, y al mismo tiempo las hogueras que se quemaban en las plazas y las calles de Petrogrado en 1917.

En 1923 en la fábrica comenzó a trabajar el famoso artista ruso Petrov-Vodkin. Hizo bocetos que luego se transfirieron a la porcelana. Su trabajo quizás más conocido es un plato titulado "La Boda". Los novios están representados en una corona de las frutas, las espigas y las flores. El plato es inseparable de la obra temprana de Petrov-Vodkin, es un estilo del icono ruso, pero mucho más divertido y colorido.

La fábrica trabajo activamente con artistas de las corrientes izquierdas: K. S. Malevich, N. M. Suetin, N. Y. Altman, I. S. Shkolnik, V. V. Lebedev, V. E. Tatlin, V. V. Kandinsky, D. P. Sterenberg. Kandinsky, antes de su partida de Rusia, hizo varios diseños para los juegos de té. Uno de los dibujos es muy figurativo: en una taza se representan los contornos de todas las iglesias de Moscú, un panorama arquitectónico inconfundible de la ciudad rusa. En otras composiciones es evidente la carga de la abstracción. Los proyectos de Kandinsky son muy decorativos y perfectamente adaptados para la pintura de porcelana. Están cerca de la estilística de sus trabajos posteriores para la Bauhaus en Weimar y Dessau. Las tazas de Kandinsky tenían una gran demanda y se han repetidoen docenas de copias. La porcelana de Kandinsky llegó a convertirse en una rareza con el tiempo. Según las publicaciones, se conocen cinco composiciones diferentes de Kandinsky en tazas y platillos guardados en museos y colecciones privadas.

\section{Suprematismo}

En 1915, Kazimir Malevich hizo el primer paso hacia la creatividad pura en el arte, pintando el lienzo con pintura negra y declarando el cuadrado como "la cara del nuevo arte" (Malevich, 2014, p. 46). Desde entonces hubo una polémica feroz acerca de esta obra, si es un fin del arte o al revés, es un comienzo del arte depurado de adornos inútiles... Y aunque la era del suprematismo duró relativamente poco, su resonancia resultó ser muy larga. Y sin duda las ideas del suprematismo tuvieron una gran influencia en los artistas de pintura de porcelana y escultores de figurines. Nikolai Suetin, el 
discípulo de Malevich, quien realizaba las ideas supremátistas en porcelana, firmaba sus trabajos con la palabra adicional "suprematismo" y añadía un sello de un cuadrado negro al lado.

El dinamismo y el drama de la era revolucionaria están plasmados en las figuras abstractas y geométricas. En esta porcelana predomina el fondo blanco e infinito de la propia porcelana. Malevich escribió: "Lo más importante en el suprematismo son dos bases: la energía de blanco y negro, que sirven para revelar la forma de la acción” (2014, p.51).

La llegada de los suprematistas se convirtió en un evento para la fábrica. Ellos estaban creando un mundo nuevo, abandonando la cultura vieja. Se puede considerar el año 1923 como crucial para la porcelana suprematista; todas las obras claves se realizaron en este mismo año. Kazimir Malevich, junto con los compañeros creaban bocetos de formas y pinturas para la fábrica. Como lo señala Kudryavtseva, Malevich es el primero en cambiar las formas de la porcelana imperial a algo "realmente novedoso" (2005, p.37).

Uno de sus proyectos más grandes fue el laboratorio de formas. En 1923 Malevich propuso organizar un taller especial dedicado a la búsqueda de nuevas formas. Los objetos creados por Malevich y sus discípulos más tarde en este laboratorio predijeron en gran medida el camino del desarrollo de la arquitectura del futuro.

Malevich actuó muy valientemente en el nuevo campo de la búsqueda artística. Él pudo descomponer la forma de los objetos condicionada por su funcionalidad hasta elementos primarios. Así la taza resultó ser de media esfera y la manija fue reemplazada por un rectángulo. Lo mismo pasa con un hervidor, es difícil de usar, pero su apariencia recuerda a Architecton de Malevich. Él se interesa más por las nuevas formas de producción en porcelana. Las formas son radicales y nacen de la conexión de diferentes volúmenes geométricos, cambiando la imagen habitual de las cosas. Malevich no trasladaba sus dibujos a los objetos de porcelana, pero lo hacían sus discípulos. Por eso se conservan muchas obras de Malevich en la porcelana realizadas por Suetin, Chashnik y otros. 
Suetin y Chashnik también estaban buscando nuevas formas de porcelana. Suetin ideó ejemplares muy interesantes. En 1923 realizó dos versiones de tinteros de bloques de estilo cubista, con dibujos suprematistas con estructuras dinámicas de rectángulos largos de tres colores principales: rojo, negro y blanco. Para 1929, Suetin descubrió varias formas nuevas: otro tintero cubista "Templo de Pskov" (llamada así porque su volúmenes estaban asociados con la estructura del templo), varios floreros y una pequeña tetera ovalada.

El trabajo activo de los suprematistas en la fábrica no duró más de un año y en 1924 los artistas fueron otras víctimas de la reforma económica de la fábrica. Suetin pasó en la fábrica más tiempo que otros suprematistas. A finales de la década de 1920, se dedicaba a la decoración de libros y al diseño. Más tarde, él colaboró en la decoración de la habitación suprematista en el Museo Ruso. En 1932, por la recomendación de la Unión de artistas de Leningrado, Suetin pasa a ser nombrado el director artístico de la fábrica de porcelana. Pero, incluso antes de este nombramiento, trabajó mucho como artista colaborador de la fábrica.

Desde el lado comercial, los trabajos de suprematistas tuvieron mucho éxito. Después del despido de los artistas que pertenecían a dicho movimiento, sus trabajos fueron copiados repetidamente por otros. Es sorprendente cómo, las cosas que no tienen valor utilitario, solamente el estético, penetraron la vida cotidiana de la gente. Incluso hoy día en la fábrica se siguen reproduciendo algunos de los diseños de mediados de los años 1920.

\section{Conclusiones}

A parte de dar a conocer la porcelana vanguardista soviética, este trabajo pretende demostrar la excepcionalidad de la misma. Detrás de todo el movimiento hubo una fuerte relación política. Pero aun así no deja de ser arte sublime y los diseños parecen más que revolucionarios incluso hoy día.

Las palabras propaganda y la porcelana parecen casi mutuamente excluyentes: propaganda es algo brutal y fuerte, cuando la porcelana es algo muy delicado y fácil de 
romper. Sin embargo, fue precisamente en porcelana donde la propaganda rusa encontró su mejor plasmacion.

\section{Recursos Bibliográficos}

Alekseev, Boris (1962), La Porcelana Artistica Sovietica 1918-1923. Moscú: Isdatelstvo Akademii Hudojestv

Andreeva, Lidiya (1975), La Porcelana Sovietica 1920-1930. Moscú: Sovetskii Hudojnik

Efros, Abraham (1930), Profiles. Moscú: Federacia

Filatov, Vladimir (1927), La Porcelana Sovietica. Moscú: Moskovskoe Hudojestevennoe Isdatelstvo

Gollerbach, Erich (1924), La Porcelana Artística Rusa. Leningrado: Gosudarstvennoe Isdatelstvo

Kudryavtseva, Tamara (2005), Alrededor del Cuadrado. San Petersburgo: The State Hermitage Publishers

Lancere, Alla (1974), La Porcelana Sovietica, Arte de la fabrica estatal de porcelana de M.V. Lomonosov. Leningrado: Hudojnik RSFSR

Malevich, Kazimir (2014), El Cuadrado Negro. San Petersburgo: Lenizdat

Sameckaya Elvira (2004), La Porcelana Sovietica de Agitación. Moscú: Mir Iskusstva 